,pdfauthor=Mohamad El Masri, Slim Abdellatif, Guy Juanole,pdfkeywords=Wireless Networks, WiFi, WiMAX, Quality of Service, Automated Highway Systems,pdfcreator=HAL,pdfproducer=PDFLaTeX,p Science $[\mathrm{cs}] /$ Networking and Internet Architecture [cs.NI]
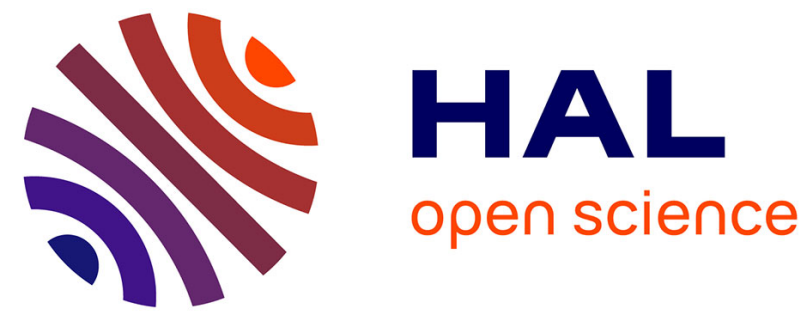

\title{
On Resource Management in Heterogeneous Wireless Access Networks application to automated highway systems
}

\author{
Mohamad El Masri, Slim Abdellatif, Guy Juanole
}

\section{To cite this version:}

Mohamad El Masri, Slim Abdellatif, Guy Juanole. On Resource Management in Heterogeneous Wireless Access Networks application to automated highway systems. NOTERE'2010. 10ème Conférence International Annuelle sur les NOuvelles TEchnologies de la REpartition, May 2010, Tozeur, Tunisia. p. 137-142. hal-00472643

\section{HAL Id: hal-00472643 https://hal.science/hal-00472643}

Submitted on 12 Apr 2010

HAL is a multi-disciplinary open access archive for the deposit and dissemination of scientific research documents, whether they are published or not. The documents may come from teaching and research institutions in France or abroad, or from public or private research centers.
L'archive ouverte pluridisciplinaire HAL, est destinée au dépôt et à la diffusion de documents scientifiques de niveau recherche, publiés ou non, émanant des établissements d'enseignement et de recherche français ou étrangers, des laboratoires publics ou privés. 


\title{
On Resource Management in Heterogeneous Wireless Access Networks application to automated highway systems
}

\author{
Mohamad El Masri*†, Slim Abdellatif*†, Guy Juanole*† \\ ${ }^{*}$ CNRS ; LAAS ; 7 avenue du colonel Roche, \\ F-31077 Toulouse, France \\ $\dagger$ Université de Toulouse ; UPS, INSA, INP, ISAE ; LAAS ; \\ F-31077 Toulouse, France \\ e-mail: \{masri,slim,juanole\}@laas.fr
}

\begin{abstract}
This paper discusses a general solution to provide quality of service (QoS) in heterogeneous wireless networks. The mechanisms we mainly discuss in this paper is resource management in a WiMAX-WiFi heterogenous wireless network within the context of an automated highway system (AHS). We show how different mechanisms within each network can be combined in order to insure the required QoS.
\end{abstract}

\section{INTRODUCTION}

The coming decades should bring, according to the most optimistic previews, a change to the way transportation works. The issue of Intelligent transportation systems goes back to the early eighties [12]. The aim of such research programs were to improve highway capacity and increase safety on the roads. Two main directions were explored in this matter. One is adapting the infrastructure to the needs by embedding magnets to the roads and highways in order to guide vehicle steering, this however proved to be highly costly and was abandoned for a more scalable solution for Automated Highway Systems (AHS): platooning (figure 1). A platoon is a formation of several vehicles logically tied on the highway, the first vehicle is called the leader of the platoon. All other vehicles within the platoon coordinate speed, acceleration and steering operations with the platoon leader. Since the vehicles are operating very closely (closer than the spacing manual driving can achieve), the highway will achieve a better throughput. This will also reduce fuel consumption and exhaust emissions by reducing aerodynamic drag. AHS will also increase safety and reduce driving stress.

In such a context, the needs in terms of communication are varied. On one level, the management of the platoons requires a communication channel to be established within each platoon (i.e. among the members of the platoon and the leader of the platoon) and between several platoons travelling on the same portion of a highway (this communication happens usually between the leaders), in some scenarios a communication channel is to be established between the platoon leaders and a central management system (informing the platoons of weather or traffic forecast for example). Another possible need of communication is entertainment-related. The driver (now

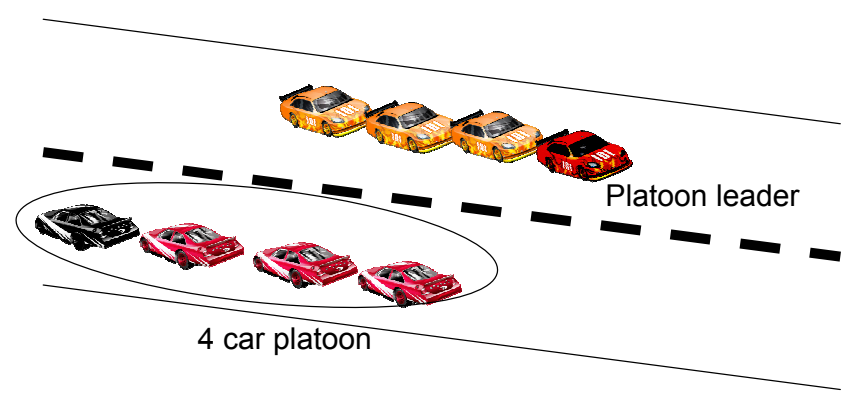

Fig. 1. Introduction to Automated Highway Systems

relaying on the system to drive the car) and those travelling with him will be in need of entertainment; the entertainment should rely on the same communication channels detailed earlier: a communication channel within the platoon, one between the platoons and one with the infrastructure. Using the same channels of communication will help reduce installation and management costs, however this will induce a severe need for Quality of Service mechanisms. The platoon management communication will need to have an enhanced Quality of Service in order to keep the platoon on track and avoid mishaps.

In this paper we will look into the different technologies that can be used within this scenario and the different mechanisms of Quality of Service to be applied. The paper is organized as follows: the second section will expose the background of this work in terms of wireless access mechanisms and quality of service in wireless access networks. The third section will give an overview of the technical access solution we advocate in such a context. The forth section will expose different quality of service mechanisms to apply in this context and the necessary mapping between those mechanisms. We will then conclude the paper and give the perspectives of this work.

\section{BACKGROUND}

We will detail in this section the different advances in terms of wireless access networks which might be interesting in the 
context of an AHS. We will look into the access mechanisms and the Quality of Service mechanisms generally used.

\section{A. Wireless access}

1) IEEE 802.11 - WiFi: IEEE 802.11 [3] is the IEEE standard specifying the physical layer and MAC sublayer enabling wireless local area access, the local area network will be formed of one Access Point (AP) and at least one station. Legacy IEEE 802.11 describes two access schemes: a CSMA/CA like distributed access scheme: DCF for Distributed Coordination Function and a polling based centralized access scheme: PCF for Point Coordination Function. Several amendments or drafts are interesting in our context:

- IEEE 802.11n [16] is an approved amendment introducing MIMO (Multiple Input Multiple Output) among other mechanisms to legacy 802.11 access thus enabling an increase in bit rates to up to $600 \mathrm{Mbits} / \mathrm{s}$.

- IEEE 802.11p [4] is the draft amendment to 802.11 enabling its use for wireless access in vehicular environment (a.k.a WAVE). The $802.11 \mathrm{p}$ work group should define the necessary mechanisms enabling a car moving at a high speed to communicate with other cars within a 1000 meter range or with roadside infrastructure.

The Quality of Service (QoS) mechanisms embedded into the 802.11 standard will be detailed in the following section.

2) IEEE 802.16 - WiMAX: IEEE 802.16 [5] is the IEEE standard specifying the physical layer and MAC sublayer enabling wireless broadband access. An IEEE 802.16 network in a point-to-multipoint mode is composed of one Base Station (BS) and at least one Subscriber Station (SS). The standard describes a connection oriented, TDM/TDMA like, medium access control scheme. This MAC scheme operates over several possible PHY layers thus giving a variety of bit rates and ranges. The most interesting amendment to the original standard in our context is the IEEE 802.16e [2] approved amendment which mainly introduced mobility related mechanisms. The QoS mechanisms that were included in the 802.16 standard will be detailed in the following section.

\section{B. Quality of Service in wireless access}

We will discuss in this section some of the efforts aiming to insure a certain Quality of Service to the users of wireless networks. We will give an overview of the mechanisms that were introduced to the standards we earlier discussed.

1) Quality of Service in 802.11: The IEEE 802.11e work group [1] introduced QoS mechanisms into the MAC layer (Medium Access Control) of the legacy IEEE 802.11 standard. This mainly consisted in the definition of a new access function: HCF (Hybrid Access Control) which combines two access modes mapped on the legacy access functions. EDCA (Enhanced Distributed Channel Access) is an enhancement of DCF and HCCA (HCF Controlled Channel Access) is an enhancement of PCF. The centralized access scheme (be it PCF or HCCA) was seldom implemented, we will thus rather look into the details of EDCA than into HCCA.
With respect to DCF, EDCA introduces the traffic differentiation concept, thus defining four access categories $(A C)$, each corresponding to a different queue within the station. A CSMA/CA like scheme is implemented by each $A C$. This scheme is based on the arbitration (characterized by the AIFS parameter (Arbitration Inter Frame Space)) and on the backoff procedure (characterized by the contention window $(\mathrm{CW})$ and the range $\left.\left[C W_{\min }, C W_{\max }\right]\right)$. The choice of AIFS and $\mathrm{CW}$ allow to prioritize the $A C$ traffic (the smaller the AIFS and $\mathrm{CW}$ range, the higher the access probability).The defined $A C \mathrm{~s}$ are, in descending priority:

- $A C_{-} V O$ : Access Category VOice,

- $A C \_V I$ : Access Category VIdeo,

- $A C_{-} B E$ : Access Category Best Effort,

- $A C \_B K$ : Access Category BacKground.

Although some $A C$ s are given an application oriented name, the mapping of applications does not have to respect this nomenclature; in fact, only the access probability changes between the different access categories. In addition to the access schemes, the work group defined several rules so as to avoid interference in access between the different concurrent access functions and thus making the different access functions respect delay constraints. The work group also introduced several frameworks enabling constructors to implement personalized QoS mechanisms. Those mechanisms are admission control for EDCA or for HCCA, traffic policing and parameters tuning.

2) Quality of Service in 802.16: WiMAX specifies four scheduling services (or Classes Of Service). Each connection is associated to a scheduling service and specifies a set of traffic and QoS parameters that qualifies its traffic's behaviour and its QoS expectations. The main considered parameters are: the traffic priority, the SDU size, the minimum reserved traffic rate, the maximum sustained traffic rate, the maximum latency or the tolerated jitter among others.

a) The requests: An uplink connection, depending on the service it is mapped to, is bound to use a set of rules specifying the way it requests bandwidth and will be served accordingly. Several methods to request bandwidth (or to specify the need to be polled) were defined by the standard:

- a unicast request opportunity is a period of airtime where only the destined connection can express its needs,

- a contention request opportunity is a period where several connections may express their needs in a CSMA/CA fashion contention based access, this kind of opportunities are programmed by the BS if, due to lack of space, it can not program enough unicast request opportunities,

- piggyback requests can be included by some connections in a specific type of headers: the Grant Management subheader,

- this same subheader contains the Poll Me bit which, if set, specifies the need of the SS to be polled for a bandwidth request by the $\mathrm{BS}$.

b) The services: The four classes of service (COS) that were defined by the WiMAX standard are: 
- Unsolicited Grant Service (UGS) : corresponds to flows with real time constraints generating the same amount of data periodically. Flows mapped to this type of service will not have to express any bandwidth request during their lifetimes. An amount of bandwidth is periodically granted to this type of flows based on the flow's specification sent out to the BS during connection establishment. Some mechanisms allow a UGS connection to either request more bandwidth to itself due to accidental map loss or to inform the BS of a poll need from non-UGS connections.

- Real-Time Polling Service (rtPS) : is used to serve flows with real time constraints having an irregular arrival profile. An rtPS connection is provided with periodic unicast request opportunities. Those opportunities will be used by the connection to express its needs depending on its queue situation.

- Non-Real-Time Polling Service (nrtPS) : is used to serve flows that have no time constraints. An nrtPS connection is provided with regular unicast request opportunities (the standard specifies an interval on the order of one second or less). An nrtPS connection can also use contention request opportunities when invited to do so.

- Best Effort (BE) : is used to serve flows with no particular needs in terms of Quality of Service. A BE connection may be granted unicast request opportunities by the BS. It may also use contention request opportunities in order to express its needs when invited to do so.

c) The grants: An SS's medium access for Uplink data transmission is done in a contention-less, polling based fashion. Within the BS, a scheduling algorithm, which is not specified in the standard, will build the UL-MAP (map of the transmission opportunities granted for the uplink direction). The UL-MAP is built based on the requests the BS received and on the initial per connection information it possesses. The requests are then granted on a per SS basis: GPSS (Grant Per Subscriber Station). In GPSS mode, transmission opportunity is granted to the SS; an uplink scheduler within the SS will then grant each of its connections a range within the granted time.

\section{WIRELESS ACCESS FOR AHS}

\section{A. Different possible solutions}

Bringing wireless access to vehicular networks is an active area of research. The main technical solution advocated is either the use of UMTS network or of 802.11 network for both inter vehicular communications [15] and vehicle to roadsideinfrastructure communications [14]. Several studies have been carried aiming to enhance the quality of wireless access. These studies revolve around several subjects like the access procedure [13], routing [10], or even achieving QoS through multi-hop routing [11].

In this paper, we will look into a more specific solution of wireless access: a heterogenous one combining the interesting distributed properties of EDCA access on a local level and the

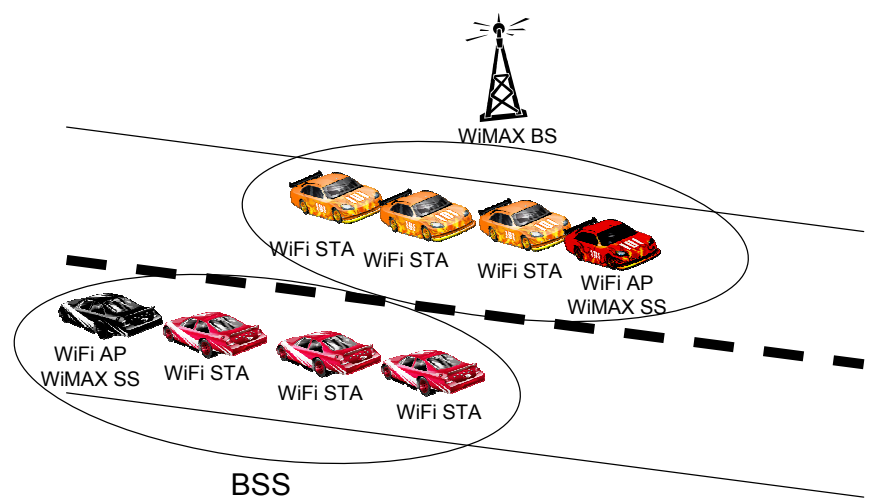

Fig. 2. The technical solution we advocate

more constrained centralized access of WiMAX on a broader level. We give the details of our solution in the following section.

\section{B. Our technical solution}

We advocate the following solution to insure the different levels of logical communication channels that the AHS application might need (figure 2)

- Each vehicle wishing to enter the AHS should be at least WiFi enabled (and to be able to act as an AP or as a simple station), this is necessary to establish intra-platoon communication. It is also recommended that a vehicle entering the system be WiMAX enabled (i.e. to have a subscriber station within it).

- Each platoon will form a WiFi BSS (Basic Service Set) on its own, the platoon leader will host the AP, each of the vehicles within the platoon will connect to its leader's BSS.

- Each platoon leader will, in addition to serving as a WIFi access point, function as a WiMAX Subscriber Station.

- WiMAX roadside infrastructure should be available on the highway. Each WiMAX BS should be configured so as to cover the largest possible area of the highway. It is also important to put in place soft handoff mechanisms within the WiMAX network.

This general architecture will enable us to insure the different communication channels:

- Intra-platoon communication is ensured through the WiFi BSS that is configured. This will allow serving both the platoon management plan and the entertainment plan. Mobility here is not an issue: since the vehicles within the platoon are all moving in the same direction and at the same speed, movement in this case is seamless. It is only when the car leaves the platoon that mobility might become an issue. However, at this level, when the car leaves the platoon, all intra platoon communications can be stopped.

- Inter-platoon communication depends on the available communication means. If both platoon leaders are 
WiMAX enabled, then Inter-platoon communication can go through the WiMAX network. If not, the platoon leaders should use the WiFi access to communicate by establishing an Extended BSS. In case of an AHS management Inter-platoon communication need, only platoon leaders will have to communicate. In case of an entertainment related communication that involves non leader vehicles, the platoon leaders will have to insure the forwarding of those communications.

- Communication with the Internet will go through the WiMAX network. The platoon leader will insure the forwarding of its subordinate vehicles communications.

\section{RESOURCE MANAGEMENT}

\section{A. QoS needs in our context}

As described above, two levels of communication needs are present in our context: the first level is critical platoon management related communications, the second level is noncritical platoon related communications (e.g. data mining) and entertainment related communications.

The critical communications will require both delay constraints and a minimum service constraint. Entertainment communications might need delay constraints and rate constraints or can be served as best effort traffic (depending on the application). Data mining traffic (and equivalent) shall be served in a Best Effort manner.

We will describe in the following sections QoS solutions that we advocate on the Local wireless access level and on the metropolitan access level. We will then give the traffic mapping that should be applied by the vehicle for its applications and on the interface level between the local and the metropolitan access.

\section{B. Admission control for EDCA}

We designed and analyzed in previous work [9] an admission control algorithm for EDCA. A general procedure of the algorithm is shown in figure 3. It goes as follows:

- General measures of the state of the medium are realized in order for the algorithm to verify if the medium would support the admission of a new flow,

- an estimation process with simple feedback correction will be applied to the measures allowing the algorithm to have a view of what the medium state would be if the new flow was to be admitted,

- this estimated state of the medium, in addition to information on active flows and flows awaiting admission are injected into a model that was designed for this purpose [8],

- the model will allow us to calculate an achievable throughput metric based on which the algorithm will give an admission decision.

The algorithm was extensively analyzed and proven to give a good protection of the flows while insuring a good utilization of the medium.

\section{Resource management for WiMAX}

We designed our resource management framework targeting the following goals: efficiency in using network resources, simplicity of the overall procedure, fairness among equivalent connections and SSs, and QoS guarantees. UGS and rtPS connections are those mainly needing QoS guarantees. We consider two QoS parameters: the "Minimum Reserved Traffic Rate" (MRTR) and "Maximum Latency". For both classes of service, we target a "Maximum Latency" of tens of milliseconds. This is a reasonable limit since the WiMax access network in our context will be a link in the global end-to-end path. Additionally, the "Minimum Reserved Traffic Rate" is guaranteed over time-scales in the order of a couple of WiMAX frame-sizes. MRTR is also guaranteed for nrtPS connections over a larger time-scale in the order of one second (as stated by the 802.16 standard).

We describe hereafter the main components of our resource management framework (the highlighted parts in figure 4) and highlight how they satisfy the above-cited objectives. The details of this framework can be found in [7].

1) A periodic and aggregate bandwidth request: Instead of having each connection request its own needs in terms of bandwidth, an aggregated request, covering all the needs of an SS is sent out to the BS periodically. This is a simpler alternative to the four bandwidth request mechanisms of the IEEE 802.16 standard and achieves better resource utilization especially under high load conditions (e.g. multicast bandwidth requests collide). Moreover, our newly designed request includes two parts : guaranteed requests and non guaranteed requests. We define the notion of contract of an SS as being the sum of the requested MRTRs of all the SS's time sensitive flows (i.e. those using UGS or rtPS types of service) that were admitted by the BS. The guaranteed bandwidth request expressed by an SS is the amount of bandwidth that have to be granted to the SS in order to have its contract fulfilled. An important feature of our proposal is to have the BS answer positively and immediately to the amount of requested bandwidth falling within the SSs contract, i.e. the grant should come in the frame following the one bearing the request. This is done in order to meet the above-cited QoS guarantees (short term MRTR and low delays). Non-guaranteed bandwidth requests are filled with nrtPS and BE connections needs. They are crucial to achieve high efficiency (and tend to a work-conserving behaviour) since they help the BS have an updated view of all the traffic awaiting at the SSs for transmission.

2) BS Scheduling : It mainly consists in granting the guaranteed bandwidth requests to the SSs. Then, sharing evenly the available bandwidth among the SSs (by accounting for their respective nrtPS connection requirements and BE connections). The operations performed by the BS are simple and done on a per SS basis (one queue and one scheduling state per SS). This results in 


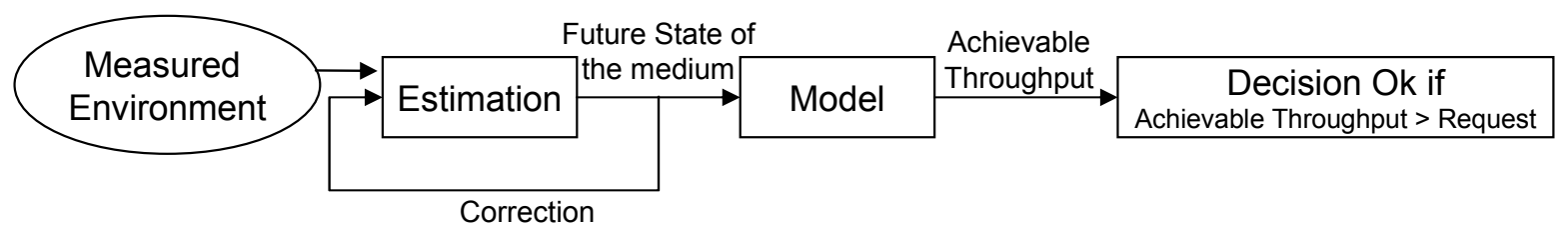

Fig. 3. Design of the admission control algorithm for EDCA

a more scalable scheduling in comparison to existing solutions that will rather adopt a scheduling algorithm on a connection basis.

3) Admission Control (AC) : Admission control is applied to each incoming connection. It is in charge of insuring that the QoS requirements of accepted connections. In other words, it checks that the guaranteed request of SSs's time-sensitive connections can be honored in the next frame and that the long-term bandwidth requirements of nrtPS connections can be satisfied. To avoid starvation, AC reserves a pre-specified portion of the bandwidth to $\mathrm{BE}$ connections. It should also have a part reserved to handle contract handoffs due to mobility.

4) SS Scheduling: SSs receive the grants on a per SS basis (GPSS). An SS shares the grant by giving priority in descending order to UGS, RT-PS, nrtPS and BE connections. The scheduling is done on a per connection basis. For nrtPS and then BE, the grants that are not used by higher priority connections are shared fairly between the active connections.

5) Policing and shaping: Some policing and shaping mechanisms are used at the SS and BS in order to tackle misbehaving connections and SSs.

SS

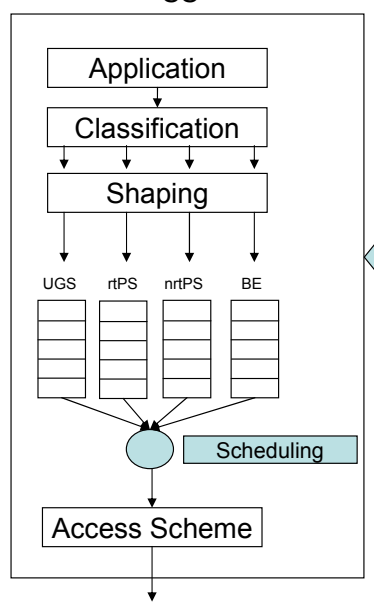

Fig. 4. General Resource management framework for WiMAX

\section{Traffic type mapping}

The following table (table I) exposes the different mappings necessary in order to serve each type of application with its required quality of service. This mapping will insure small

\begin{tabular}{|l|c|c|}
\hline Application type & EDCA $A C$ & WiMAX COS \\
\hline Platoon management (critical) & $A C_{-} V O$ & UGS or rtPS \\
\hline Entertainment (time constrained) & $A C_{-} V I$ & UGS or rtPS \\
\hline Entertainment (non real time) & $A C_{-} B E$ & nrtPS \\
\hline Platoon related (non critical) & $A C_{-} B K$ & BE \\
\hline
\end{tabular}

TABLE I

MAPPING THE APPLICATIONS

delays for local access within the platoon when necessary and will give guarantees on inter-platoon communications using our resource management framework. It will also protect these critical communications from non critical, yet QoS requiring, traffic. A good QoS will be serviced to entertainment traffic. We give here a general set of rules to be applied in the nominal case. In case WiMAX communication is not available, critical platoon management communications should use EDCA's $A C_{-} V O$ for inter-platoon communications.

\section{CONCLUSION}

We exposed in this paper a general view of a QoS solution for heterogenous wireless access networks. The solution we advocate is here put in the context of an automated highway system. Our solution is composed of an admission control algorithm for the local wireless access and a general resource management framework for the metropolitan wireless access. Both solutions have been proven to achieve the required QoS on a stand-alone basis. One might argue that the technologies which we advocate using in our solution will become obsolete before Automated Highway Systems become reality. This might be true, however the paradigms and general mechanisms will be the same even with an enhanced technology. In this context, the QoS solutions we detailed in this paper will also be entirely adaptable to the new technologies.

This work is being extended and further analyzed. We will look into applying AHS specific mobility models as those exposed in [6] in order to have a correct view of the effects of mobility on our solution. Another possible axis of study is to analyze the performance of integrating 802.11p mechanisms into our architecture. 


\section{REFERENCES}

[1] "Ieee standard for information technology - telecommunications and information exchange between systems - local and metropolitan area networks - specific requirements part 11: Wireless lan medium access control (mac) and physical layer (phy) specifications amendment 8: Medium access control (mac) quality of service enhancements," IEEE Std 802.11e-2005 (Amendment to IEEE Std 802.11, 1999 Edition (Reaff 2003)), pp. 0-189, 2005.

[2] IEEE Standard for Local and Metropolitan area Networks Part16 : Air Interface for fixed broadband Wireless Access Systems - Amendment 2: Physical and Medium Access Control Layers for Combined Fixed and Mobile Operation in Licensed Bands, 2005.

[3] "Ieee standard for information technology-telecommunications and information exchange between systems-local and metropolitan area networks-specific requirements - part 11: Wireless lan medium access control (mac) and physical layer (phy) specifications," IEEE Std 802.112007 (Revision of IEEE Std 802.11-1999), pp. C1-1184, Dec. 2007.

[4] "Ieee draft standard for information technology - telecommunications and information exchange between systems - local and metropolitan area networks -specific requirements - part 11: Wireless lan medium access control (mac) and physical layer (phy) specifications amendment 7: Wireless access in vehicular environments," Draft Std P802.11p, May 2009.

[5] "Ieee standard for local and metropolitan area networks part 16: Air interface for broadband wireless access systems," IEEE Std 802.16-2009 (Revision of IEEE Std 802.16-2004), pp. C1-2004, May 2009.

[6] O. Hamouda, M. Kaaniche, E. Matthiesen, J. Rasmussen, and H.P. Schwefel, "Connectivity dynamics in vehicular freeway scenarios," The 2nd IEEE International Workshop on ITS for Ubiquitous Roads (UBIROADS'2009), June 2009.
[7] M. E. Masri, S. Abdellatif, and G. Juanole, "An uplink bandwidth management framework for ieee 802.16 with qos guarantees," 8th International IFIP-TC6 Networking Conference, NETWORKING 2009, May 2009.

[8] M. E. Masri, G. Juanole, and S. Abdellatif, "A synthetic model of ieee 802.11e edca," International Conference on Latest Advances in Networks, ICLAN '07, Dec. 2007.

[9] — - "Hybrid admission control algorithm for ieee 802.11e edca: analysis," International Conference on Networks, ICN '08, April 2008.

[10] Z. Niu, W. Yao, Q. Ni, and Y. Song, "Study on qos support in 802.11ebased multi-hop vehicular wireless ad hoc networks," IEEE International Conference on Networking, Sensing and Control 2007, April 2007.

[11] C. L. Ramrez and M. F. Veiga, "Oos in vehicular and intelligent transport networks using multipath routing," IEEE International Symposium on Industrial Electronics, 2007. ISIE 2007., June 2007.

[12] S. Shladover, "Path at 20 - history and major milestones," in Intelligent Transportation Systems Conference, 2006. ITSC '06. IEEE, Sept. 2006, pp. 1_22-1_29.

[13] H. Su and X. Zhang, "Cross-layer based opportunistic mac protocols for qos provisionings over cognitive radio wireless networks," IEEE Journal on Selected Areas in Communications, vol. 26, no. 1, Jan. 2008.

[14] D. Valerio, F. Ricciato, P. Belanovic, and T. Zemen, "Umts on the road: Broadcasting intelligent road safety information via mbms," IEEE Vehicular Technology Conference, 2008. VTC Spring 2008., May 2008.

[15] C. Wewetzer, M. Caliskan, K. Meier, and A. Luebke, "Experimental evaluation of umts and wireless lan for inter-vehicle communication," 7th International Conference on ITS Telecommunications, 2007. ITST '07., June 2007.

[16] Y. Xiao, "Ieee 802.11n: enhancements for higher throughput in wireless lans," Wireless Communications, IEEE, vol. 12, no. 6, pp. 82-91, Dec. 2005. 